\title{
Aspects of European Argyra systematics: molecular insights and morphology (Diptera: Dolichopodidae)
}

\author{
Christoph Germann, Marc Pollet \& Marco Valerio Bernasconi*
}

\begin{abstract}
Germann, C., Pollet, M. \& Bernasconi, M. V. 2011: Aspects of European Argyra systematics: molecular insights and morphology (Diptera: Dolichopodidae). Entomol. Fennica 22: 5-14.

We focused on systematic aspects of some of the most common European Argyra species. Bayesian and Maximum Parsimony analyses were performed, using three mitochondrial markers (COI, 12S, and 16S) with ten Argyra species as the ingroup, and three other Diaphorinae as an outgroup. The topology of the trees derived from the two analyses was slightly different but not conflicting and allowed the identification of five species groups corroborated by morphological characters. The Argyra argyria species group and the Argyra atriceps species group encompass the majority of the species and can be regarded as Argyra sensu stricto. The Argyra diaphana species group is characterized by a pubescent scutellum, a characteristic of Lasiargyra. The Argyra elongata species group has previously always been regarded as Argyra s.s., but consistently clustered separately from this clade in our analyses. The Argyra vestita species group corresponds with Leucostola, and is characterized by a bare scape.

C. Germann \& M. V. Bernasconi (*corresponding author), Zoological Museum, Institute of Evolutionary Biology and Environmental Studies, University of Zurich, Winterthurerstrasse 190, CH-8057 Zurich, Switzerland; E-mail: *marco.bernasconi@access.uzh.ch

M. Pollet, Research Group Terrestrial Ecology (TEREC), Department of Biology, Ghent University, K.L. Ledeganckstraat 35, B-9000 Ghent, Belgium; Department of Entomology, Royal Belgian Institute of Natural Sciences (KBIN), Vautierstraat 29, B-1000 Brussels, Belgium; and Information and Data Center (IDC), Research Institute for Nature and Forest (INBO), Kliniekstraat 25, B1070 Brussels, Belgium
\end{abstract}

Received 17 March 2010, accepted 28 May 2010

\section{Introduction}

Argyra is a primarily Holarctic genus with 100 species worldwide (Yang et al. 2006; see also Sinclair et al. 2008). Its species diversity is comparable in the Nearctic (46 sp.; Pollet et al. 2004) and the Palaearctic (42 sp.; Negrobov 1991, Pollet 2007, Selivanova \& Negrobov 2006a, 2006b,
2007) with only a few known representatives from the Neotropical and the Oriental realm. Argyra is represented by 26 species in Europe (Pollet 2007).

All species of the genus Argyra are characterized by (i) a vertical row of 2 or more erect setae on the hind coxa, decreasing progressively in length towards the apex, with the uppermost one 
inserted near the basis of the coxa. Other features are equally diagnostic and found in most species: (ii) the silvery pruinosity of thorax and abdomen (males), and (iii) a scape with a dorsal pubescence (bare in Leucostola). The following features are encountered in many Argyra species, but are not unique to this genus: (iv) a concave occiput, (v) a rather large head closely attached to the thorax, (vi) distinctly pubescent eyes, (vii) a prothoracic spiracle bordered with dense pubescence, (viii) biserial acrostichal setae, (ix) six dorsocentral setae, (x) four scutellar setae (see further), (xi) anterior abdominal segments often yellow or with yellow lateral patches, (xii) hypopygium often with macrochaetae, and (xiii) hind femur without preapical seta (except in A. elongata). The general structure of the hypopygium is characteristic as well: the hypopygium itself is entirely symmetrical; the hypandrium is simple, shows a ventral curve and largely encloses the aedeagus with an enlarged apex; the basiventral epandrial lobe is reduced and represented by 2 or more epandrial setae; the apicoventral epandrial lobe is usually well developed and equal-sized to the surstylus; the surstylus is composed of an inner and outer plate, the latter showing a blunt apex and marginal setae, most of which are directed towards the inner side; the cercus is usually rather small, triangular with strong outer setae.

Although representatives of Argyra are easily and unequivocally recognizable, in the past several attempts have been made to split off species in subgenera or even separate genera, e.g. species with a pubescent scutellum - a characteristic trait of Lasiargyra Mik, 1878 - and a bare scape, typical for Leucostola Loew, 1857. In addition, Bernasconi et al. (2007b) speculated on a separate position of Argyra elongata in respect of the other Argyra species. In the following we present and discuss new insights into the systematic positions of some common European Argyra species.

\section{Material and methods}

\subsection{Samples}

A total of 16 specimens of 13 species of European Diaphorinae (Dolichopodidae) were included in the present study, with 10 Argyra species (12 specimens) as the ingroup, and 3 species (4 specimens) of 3 other diaphorine genera (Asyndetus latifrons (Loew, 1857), Diaphorus nigricans Meigen, 1824, Chrysotus neglectus (Wiedemann, 1817)) as an outgroup (Table 1). Outgroup species were selected on the basis of a previous phylogenetic analysis (Bernasconi et al. 2007b). All samples are conserved in $100 \%$ ethanol at $20^{\circ} \mathrm{C}$.

\subsection{DNA extraction, amplification, and sequencing}

DNA was extracted from fly specimens using a Dneasy Tissue kit (Qiagen AG, Hombrechtikon, Switzerland) carefully following the manufacturer's instructions (for more details, see Bernasconi et al. 2007a, 2007b). Standard PCR reactions and subsequent direct sequencing (including amplification and sequencing primers, Microsynth $\mathrm{GmbH}$, Balgach, Switzerland) were performed following the methods reported in details in Germann et al. (2010).

\subsection{DNA sequence analyses}

The mitochondrial sequences (COI, 12S, and 16S) were handled and stored with the Lasergene program Editseq (DNAstar Inc., Madison, WI USA). Alignment of all gene sequences was performed using Megalign (DNAstar Inc.) with default multiple alignment parameters ("gap penalty $=15$ "; "gap length penalty $=6.66 "$ " "delay divergent sqs $(\%)=30$ "; "DNA transition weight $=$ 0.50 "). The COI alignment included a single gap of three nucleotides (caused by the deletion recorded in Diaphorus nigricans Meigen and Asyndetus latifrons Loew, before the stop codon; see also Bernasconi et al., 2007b, Pollet et al., 2010). Concerning the $16 \mathrm{~S}$ and $12 \mathrm{~S}$ gene fragments, the obtained alignments using default values were not subsequently manually adjusted. In the phylogenetic analyses, gaps were treated as "missing". Phylogenetic reconstruction was carried out using Bayesian analysis (BAY), performed with MrBayes version 3.1.2 (Ronquist \& Huelsenbeck 2003) and Maximum Parsimony (MP; using the heuristic search with stepwise ad- 
Table 1. Species (samples) used in the present study with the respective GenBank accession numbers.

\begin{tabular}{|c|c|c|c|c|}
\hline \multirow[t]{2}{*}{ Species } & \multirow{2}{*}{$\begin{array}{l}\text { Origin of specimen } \\
\text { Belgian province: locality }\end{array}$} & \multicolumn{3}{|c|}{ GenBank accession number } \\
\hline & & $\mathrm{COI}$ & $12 S$ rDNA & 16S rDNA \\
\hline \multicolumn{5}{|l|}{ Argyra (ingroup) } \\
\hline Argyra argentina (Meigen, 1824) & Oost-Vlaanderen: Aalst & DQ456922 & DQ464766 & GU827369 \\
\hline Argyra argyria (Meigen, 1824) & Oost-Vlaanderen: Denderhoutem & DQ456888 & DQ464767 & GU827370 \\
\hline Argyra atriceps Loew, 1857 & Oost-Vlaanderen: Denderhoutem & DQ456890 & DQ464819 & GU827371 \\
\hline Argyra atriceps & Oost-Vlaanderen: Aalst & DQ456924 & DQ464769 & GU827372 \\
\hline Argyra diaphana (Fabricius, 1775) & Oost-Vlaanderen: Denderhoutem & DQ456884 & DQ464865 & GU827373 \\
\hline Argyra elongata (Zetterstedt, 1843) & Hainaut: Chimay, Lac de Virelles & GU827382 & $\mathrm{NA}^{*}$ & GU827374 \\
\hline Argyra grata Loew, 1857 & Limburg: Sint-Pieters-Voeren & DQ456934 & DQ464782 & GU827375 \\
\hline Argyra ilonae Gosseries, 1988 & Oost-Vlaanderen: Aalst & GU827383 & NA & GU827376 \\
\hline Argyra leucocephala (Meigen, 1824) & Oost-Vlaanderen: Denderhoutem & DQ456883 & DQ464859 & EU864019 \\
\hline Argyra perplexa Becker, 1918 & Oost-Vlaanderen: Aalst & DQ456923 & DQ464768 & GU827377 \\
\hline Argyra vestita (Wiedemann, 1817) & Oost-Vlaanderen: Baasrode & DQ456939 & DQ464794 & GU827378 \\
\hline Argyra vestita & Oost-Vlaanderen: Denderleeuw & DQ456945 & DQ464802 & GU827379 \\
\hline \multicolumn{5}{|l|}{ Other Diaphorinae (outgroup) } \\
\hline Asyndetus latifrons (Loew, 1857) & Antwerpen: Nijlen & GU827384 & NA & GU827380 \\
\hline Chrysotus neglectus (Wiedemann, 1817) & Limburg: Zonhoven & DQ456893 & DQ464829 & EU864020 \\
\hline Chrysotus neglectus & Antwerpen: Nijlen & GU827385 & NA & GU827381 \\
\hline Diaphorus nigricans Meigen, 1824 & Limburg: Zonhoven & DQ456894 & DQ464833 & EU864021 \\
\hline
\end{tabular}

*Abbreviations: NA: not available.

dition option, TBR - Tree Bisection Reconnection - branch swapping, and 100 additional replicates) using PAUP*4.0b10 (Swofford 2002); reliability of internal branches for the Maximum Parsimony analysis was assessed by bootstrapping with 5,000 pseudo-replicates. Modeltest 3.5 (Posada \& Crandall 1998) enabled us to identify the evolutionary model(s) for the Bayesian analyses. Data were partitioned by gene (COI, $12 \mathrm{~S}$, and $16 \mathrm{~S}$ ) and the COI gene was further partitioned by codon (first-, second-, and third-codon position). Bayesian analyses were allowed to use a mixed model (i.e. a model in which all genes have their unique GTR $+\mathrm{I}+\mathrm{G}$ model) and the Markov chain Monte Carlo search was run with 4 chains (one cold and three heated) for 1,000,000 generations, with trees being sampled every 100 generations. To determine the "burn-in", log-likelihood plots were examined for stationarity (where plotted values reach an asymptote). Stationarity was clearly reached already after less than 100,000 generations; thus we discarded the first 1,000 trees. Higher "burn-in" did not alter the topology of the final $50 \%$ majority rule consensus tree(s). Bayesian posterior probabilities were therefore given by the percentage of runs that produced each branch and were calculated from the remaining trees generated from the two parallel runs. The two independent runs executed in parallel, converged very fast, reaching average standard deviation values of the split frequencies of less than 0.05. MEGA (Molecular Evolutionary Genetics Analysis version 4.0; Tamura et al. 2007) was also used for managing and drawing the trees produced by the various phylogenetic analyses and to calculate the genetic distances. The sequences of the three mitochondrial genes for the 16 Diaphorinae specimens analysed here have been deposited in GenBank (Table 1).

\section{Results}

\subsection{Molecular phylogeny, overview}

Preliminary phylogenetic analyses were performed including all 16 specimens (Table 1). As all specimens of each species made monospecific clades, one single specimen of each species was randomly selected for incorporation into the final analysis. All results presented here are therefore based on the total molecular evidence resulting 


\section{Bayesian analysis}

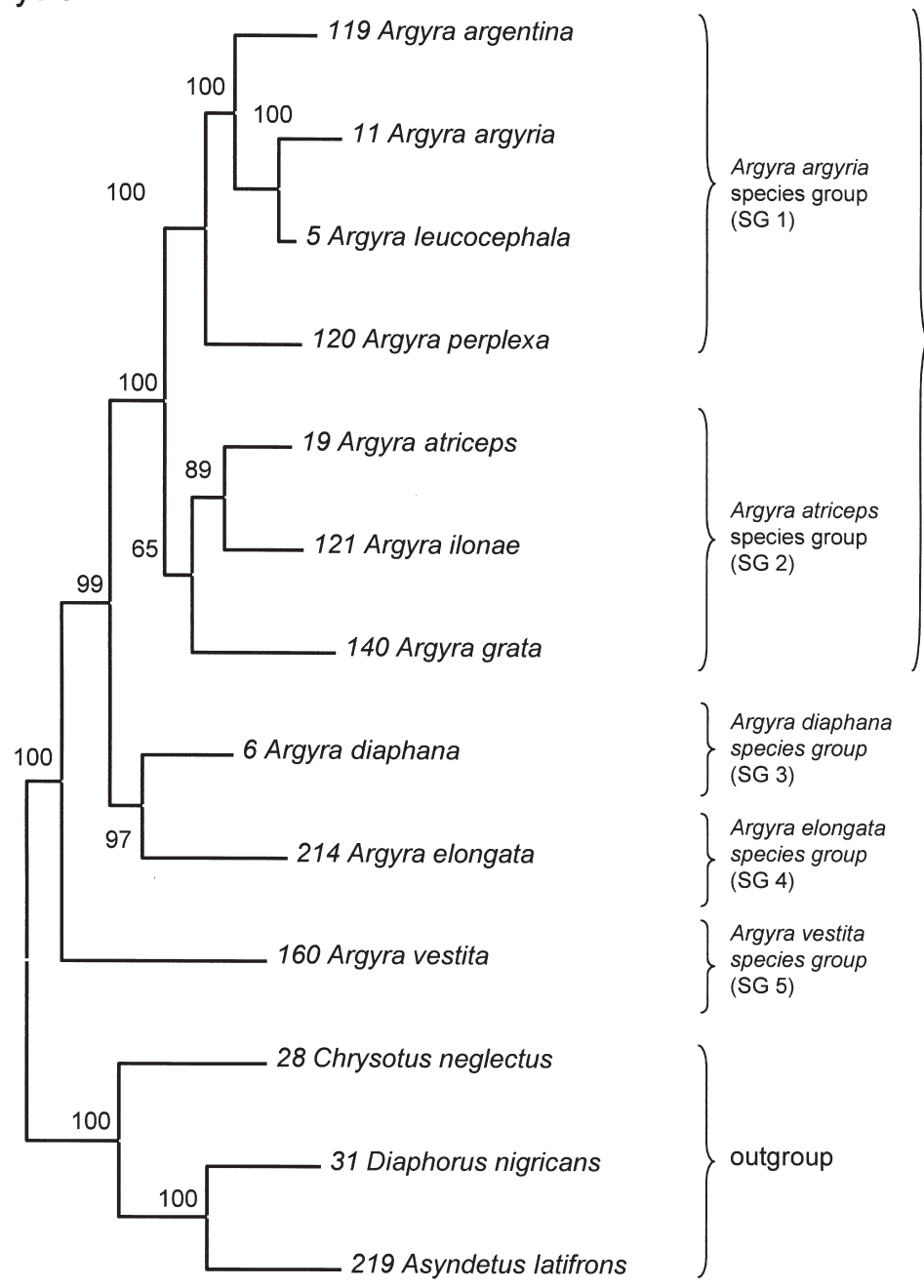

$\overline{0.05}$

from the concatenation of the three mitochondrial genes. The full data set comprises 1713 characters (COI: 810; 12S: 372; 16S: 531) with 561 variable sites (COI: 271; 12S: 133; 16S: 157) and 364 parsimony informative sites (COI: 200; 12S: 60; 16S: 104).

Phylogenetic relationships derived from 18,002 Bayesian trees $(9,001$ trees for each of the two parallel runs) based on all combined sequences and 13 diaphorine species are illustrated in Fig. 1. The Maximum Parsimony analysis (Fig. 2) produced a single most parsimonious tree of length 1281 (consistency index $=0.564$; retention index $=0.432$; rescaled consistency index $=$ 0.244 ; homoplasy index $=0.436$ ). The topology of both trees appears to be slightly different but not conflicting (see further). Hereafter, Bayesian analysis refers to the tree in Fig. 1, and Maximum Parsimony analysis to that in Fig. 2; value of posterior probabilities in the Bayesian analysis and of the bootstrap support in the Maximum Parsimony analysis are abbreviated as "pp" and "bs", respectively.

Both the Bayesian and Maximum Parsimony analyses revealed 5 species groups within the genus that can be characterized as follows: 


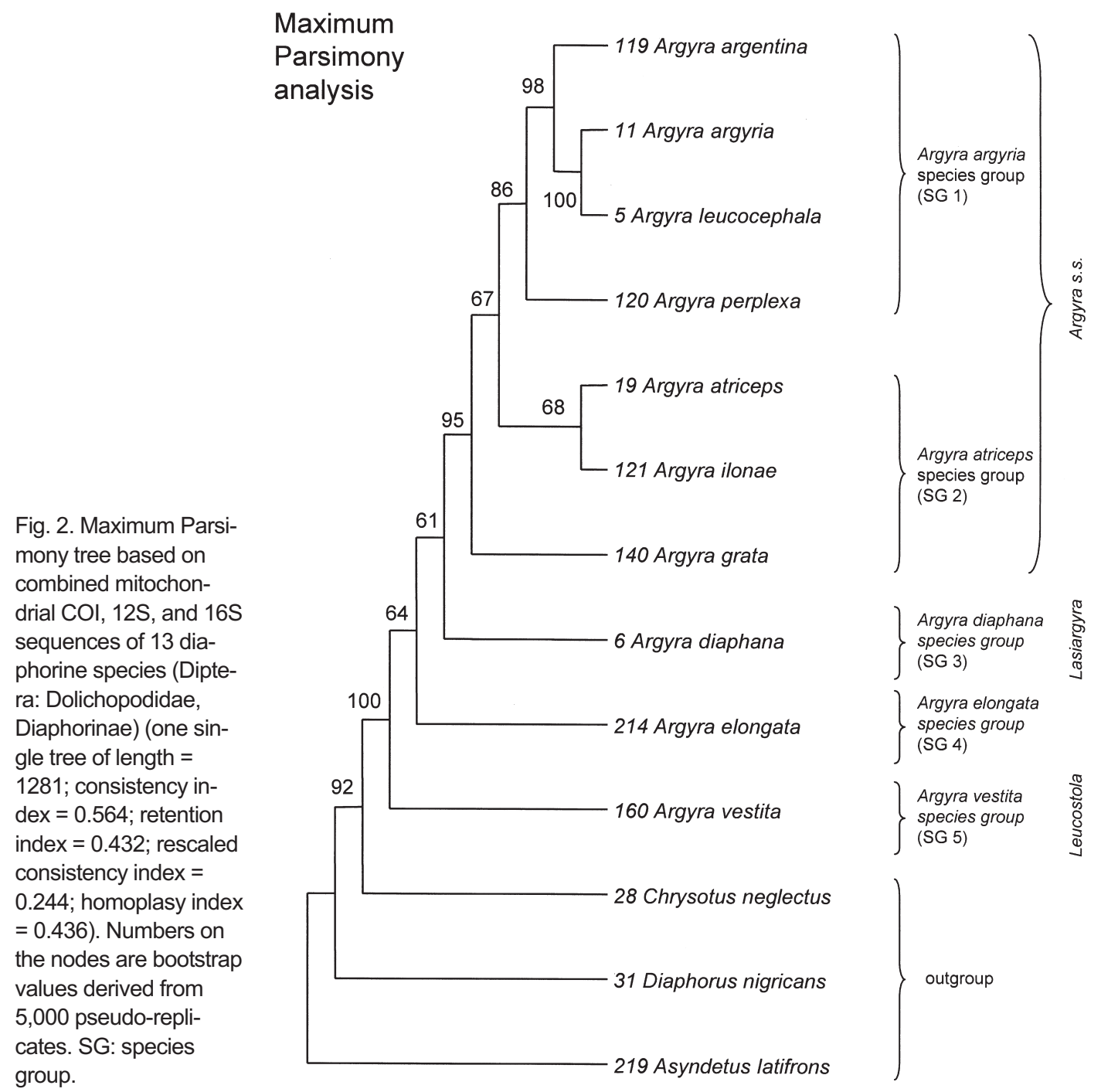

\subsection{Argyra argyria species group (SG 1), and Argyra atriceps species group (SG 2)}

The combined clade of SG 1 and SG 2 is strongly supported in both analyses (BAY: 100\% pp; MP: 95\% bs), as well as SG 1 (BAY: 100\% pp; MP: $86 \% \mathrm{bs}$ ), and corresponds roughly with the concept of Argyra sensu stricto. All species of this combined clade share the same general habitus and feature a pubescent scape (Fig. 3f), bare scutellum (Fig. 3d), and a hind femur without preapical setae. Nevertheless, the genetic distance between species of SG 1 and SG 2 is on average as large as between species of the combined SG 1-SG 2 and A. diaphana, and only somewhat smaller than between the species of the combined SG 1-SG 2 on the one hand, and A. elongata and A. vestita on the other hand (Table 2), which is also reflected in morphological traits (see further).

All species of SG 1 are characterized by a mesonotum with a silvery pruinosity and the lack of macrochaetae on the genital capsule (Fig. 3a). Their abdomen also features a similar strong silvery gloss, but this is also found in A. ilonae Gosseries, 1988, A. diaphana and $A$. vestita. The species clade including $A$. argyria and $A$. leucocephala (Meigen, 1824) is strongly supported in both analyses (both 100\%). Both species share multiple postocellar setae, a postpedicel that is at 
Table 2. Overview of the genetic distances (uncorrected p-distance) between all Argyra and outgroup species for the combined data set (COI, 12S, and $16 \mathrm{~S}$ sequences).

\begin{tabular}{|c|c|c|c|c|c|c|c|c|c|c|c|c|}
\hline & {$[1]$} & [2] & [3] & [4] & [5] & [6] & [7] & [8] & [9] & [10] & [11] & [12] \\
\hline \multicolumn{13}{|c|}{$\begin{array}{l}\text { Argyra (ingroup) } \\
\text { [1] }\end{array}$} \\
\hline [2] & 0.10 & & & & & & & & & & & \\
\hline [3] & 0.11 & 0.11 & & & & & & & & & & \\
\hline [4] & 0.12 & 0.11 & 0.11 & & & & & & & & & \\
\hline [5] & 0.12 & 0.12 & 0.11 & 0.09 & & & & & & & & \\
\hline [6] & 0.12 & 0.12 & 0.10 & 0.12 & 0.11 & & & & & & & \\
\hline [7] & 0.11 & 0.11 & 0.08 & 0.11 & 0.11 & 0.10 & & & & & & \\
\hline [8] & 0.08 & 0.06 & 0.10 & 0.10 & 0.11 & 0.11 & 0.10 & & & & & \\
\hline [9] & 0.11 & 0.10 & 0.10 & 0.11 & 0.12 & 0.12 & 0.10 & 0.09 & & & & \\
\hline$[10]$ & 0.16 & 0.15 & 0.14 & 0.12 & 0.12 & 0.14 & 0.13 & 0.14 & 0.14 & & & \\
\hline \multicolumn{13}{|c|}{ Other Diaphorinae (outgroup) ${ }^{\S}$} \\
\hline [11] & 0.15 & 0.14 & 0.15 & 0.14 & 0.13 & 0.15 & 0.15 & 0.14 & 0.15 & 0.15 & & \\
\hline [12] & 0.15 & 0.14 & 0.14 & 0.13 & 0.12 & 0.14 & 0.13 & 0.13 & 0.14 & 0.14 & 0.11 & \\
\hline [13] & 0.15 & 0.15 & 0.15 & 0.14 & 0.13 & 0.15 & 0.13 & 0.14 & 0.15 & 0.14 & 0.10 & 0.11 \\
\hline
\end{tabular}

\# Ingroup: [ 1] Argyra argentina; [ 2] A. argyria; [ 3] A. atriceps; [ 4] A. diaphana; [ 5] A. elongata; [ 6] A. grata; [ 7] A. ilonae; [ 8] A. leucocephala; [ 9] A. perplexa; [10] A. vestita.

§Outgroup: [11] Asyndetus latifrons; [12] Chrysotus neglectus; [13] Diaphorus nigricans.

most $1.5 \times$ as long as wide, and a black face and frons, three characters with different states in $A$. argentina (Meigen, 1824) and A. perplexa Becker, 1918.

All species of SG 2 share macrochaetae (of varying sizes) on the genital capsule (Fig. 3b), and the lack of a silvery pruinosity of the mesonotum. In addition, both $A$. atriceps Loew, 1857 and A. ilonae have the hind metatarsus longer than the $2^{\text {nd }}$ tarsomere of the hind leg, and a black face and frons, two characters not present in the largely pale $A$. grata Loew, 1857 . The genetic distance between the species of SG 2 is similar to that among the SG1 species (see Table 2).

\subsection{Argyra diaphana species group (Lasiargyra) (SG 3) and A. elongata species group (SG 4)}

The $A$. diaphana species group is characterized by a pubescent scutellum (Fig. 3c), and thus corresponds with the currently invalid taxonomic concept of Lasiargyra. Its sister clade position to Argyra s.s. (SG 1-SG 2) is strongly supported in both analyses (BAY: 99\% pp; MP: 95\% bs), but the phylogenetic relationship with $A$. elongata is less certain (only supported in BAY: 97\% pp). And although the genetic distance $(0.088)$ between A. diaphana and A. elongata is comparable with that between species within SG1 and SG2, it is not supported by shared morphological traits, unique to each of the species (groups). $A$. elongata is the only species thus far described with a strong preapical seta on the hind femur. Apart from that, it differs from all other species by the distinct robustness of its legs, the strong tibial setae, the lack of fine ventral setae on the femora, and the two large erect setae on the hind coxa (versus 4-7 in all other species, except $A$. vestita). Further more, the apicoventral epandrial lobe (hypopygium) is largely merged with the surstylus, which is not the case in the other investigated species.

\subsection{Argyra vestita species group (Leucostola) (SG 5)}

This species group can be considered the sister clade to the remaining Argyra species as confirmed by strong support in both analysis. This clade is mainly supported by the lack of pubescence of the scape (Fig. 3e). The average genetic 

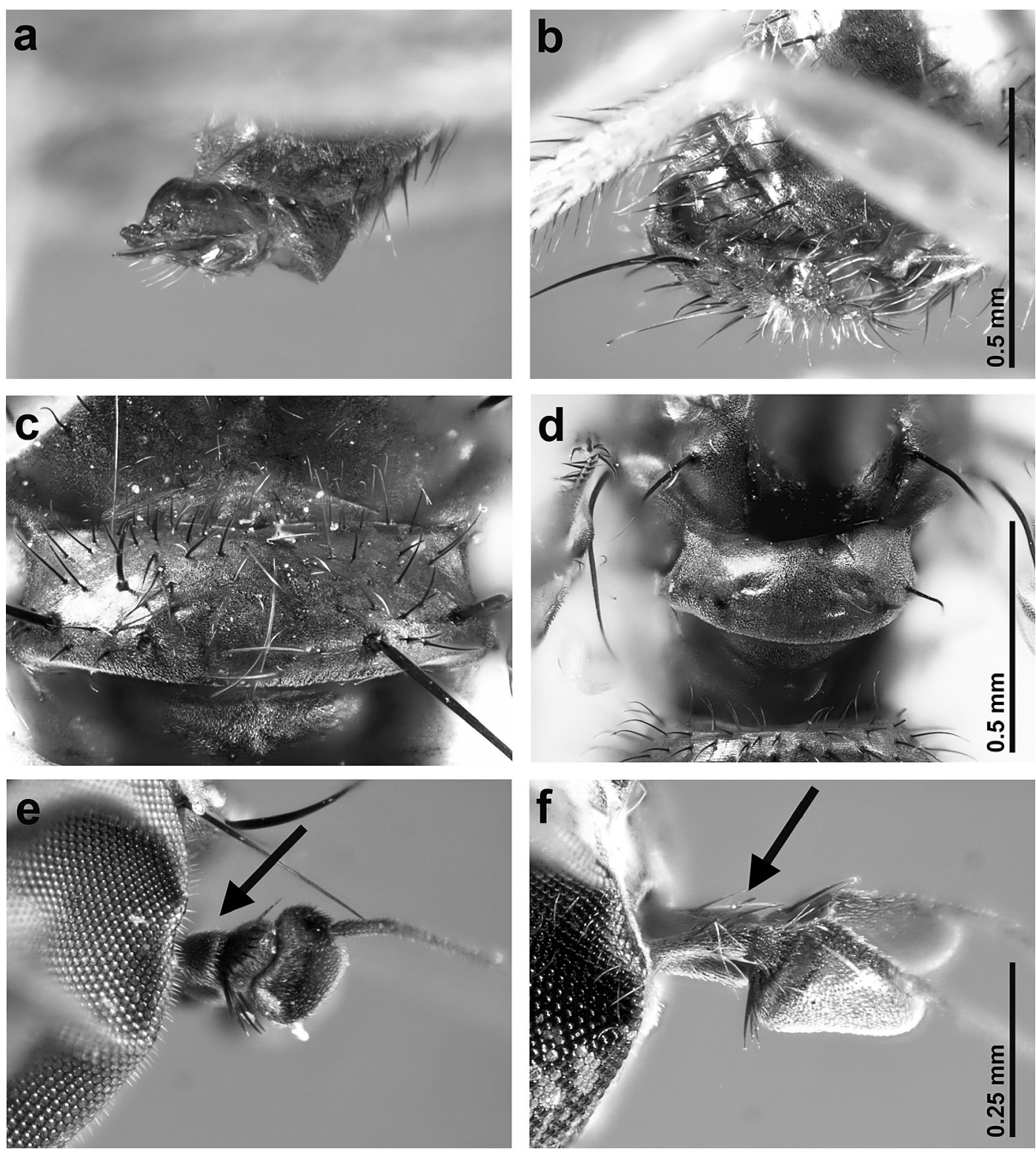

Fig. 3. - a. Argyra argentina (SG 1), genital capsule without macrochaetae. - b. A. grata (SG 2), genital capsule with macrochaetae. - c. A. diaphana (SG 3), pubescent scutellum. - d. A. grata (SG 2), bare scutellum. - e. A. vestita (SG 5), bare scape. - f. A. grata (SG 2), pubescent scape.

distance between $A$. vestita and the remaining nine Argyra species is 0.137 , comparable with the somewhat lower average distance between $A$. elongata and the other Argyra species (0.113), and both are lower than the average distance between all Argyra species and the three outgroup species (0.158).

\section{Discussion}

\subsection{Argyra sensu stricto (SG1 and SG2)}

The molecular trees presented here enabled us to gain some insights into the intrageneric structure of European species of the genus Argyra. We discuss these findings including a number of sup- 
portive morphological traits. Within Argyra s.s., the presence or absence of a silvery pruinosity of the mesonotum, and of macrochaetae on the genital capsule might explain the break-up in two separate clades (SG1 and SG2, Figs $1 \& 2$ ). As macrochaetae are also present in A. diaphana, $A$. elongata and $A$. vestita, their absence in Argyra SG 1 might further be hypothesized as secondary loss.

\subsection{Argyra diaphana species group (SG3) - Lasiargyra}

The scutellar pubescence is a character of debate since its application to assign species with a pubescent scutellum to Lasiargyra by Mik (1878). This taxonomic name has been largely ignored (Lundbeck 1912) or treated as a synonym of Argyra (Becker 1918, Parent 1938). In the present study, $A$. diaphana does not make part of Argyra s.s., but interestingly, its average genetic distance with species of SG 1-SG 2 is almost identical to the average distance between species of SG1 and SG2. Our results provide a first hint to a position of Lasiargyra outside Argyra s. s. However, obviously a broader species sample is needed to elaborate the use of the subgeneric status of Lasiargyra.

\subsection{Argyra elongata species group (SG 4)}

In contrast to the two previous lineages, surprisingly enough, $A$. elongata has never been considered as representative of a separate (subgeneric) lineage despite a number of distinct features e.g. the preapical seta of the hind femur. Although Lundbeck (1912), Becker (1918) and Parent (1938) mentioned this feature, only Assis Fonseca (1978) actually used it in his identification key. Like the pubescence of the scape (see further down), also the presence of a preapical seta on the hind femur plays an important diagnostic role in the delimitation of some subfamilies (e.g. absent in all Neurigoninae and Medeterinae) and genera (e.g. present in Sciapus in contrast to other Sciapodinae). Its phylogenetic relevance, though, seems more marginal at other taxonomic levels. E.g. Rhaphium Meigen, 1803 encompasses both species with and without a preapical seta of the hind femur, whereas in the male of Achalcus vaillanti Brunhes, 1987 a secondary loss is observed (Pollet 1996). The position of $A$. elongata outside the Argyra s.s. clade (average genetic distance with Argyra SG 1-SG 2: 0.115) might be explained by the deviating morphology. The strongly supported clade in the Bayesian analysis with $A$. diaphana, however, cannot be corroborated from a morphological perspective. Nevertheless, the formerly speculated position outside an Argyra s.s. clade by Bernasconi et al. (2007b) is confirmed with the present results.

\subsection{Argyra vestita species group (SG5) - Leucostola}

The systematic value of the dorsal pubescence of the scape in Argyra, or rather the lack of it, has been the subject of much confusion in the past. The bare scape of $A$. vestita convinced Loew (1857) to erect the genus Leucostola. He based his decision on the fact that this had been a key feature in separating entire subfamilies (e.g. present in all Dolichopodinae) or genera (Syntormon versus Parasyntormon in Sympycninae). Although many contemporary and later authors recognized Leucostola as a valid genus (e.g. Lundbeck 1912), some of them appeared clearly less convinced and tried to find additional features to support the separate position of this taxon. Kowarz (1879) added the poor ventral chaetotaxy of the mid tibia in A. vestita as diagnostic (against a spacy serration in the other species) which has, indeed, been observed in specimens of $A$. vestita examined by the authors, and is also mentioned in the original description of $A$. miki (Kowarz, 1882). Becker (1918) sank Leucostola to subgeneric level but mentioned the presence of only two strong scutellar setae as supplementary character. This character, however, is not considered sufficiently unequivocal: the lateral pair of setae is, indeed, substantially smaller than the medial pair in $A$. vestita, but a clear size difference has also been established in species of SG 1 ( $A$. perplexa) and SG 2 (A. ilonae). In his revision of the North American Argyra species, Van Duzee (1925) even mentioned that the pubescence of the scape strongly dwindles in value and is merely 
represented by one single seta in some species. Nevertheless, he also recognized Leucostola as subgenus of Argyra. Parent's (1938) treatment of Leucostola is ambiguous as it is both indicated as separate genus and as subgenus of Argyra. This author already highlighted that the scutellar setae are not reliable as diagnostic character. In fact, some species of Argyra s.s. (A. biseta Parent, 1929) even show a single pair of scutellar setae (i.e. with a total loss of the lateral pair). The morphological basis for the (sub)generic status of this taxon thus is rather weak, hence the fact that it was not recognized as such by Shiner (1862). It has been treated likewise in the most recent catalogues (Negrobov 1991, Pollet et al. 2004, Yang et al. 2006; see also Sinclair et al. 2008). The present study indicates a separate position of $A$. vestita (see Table 2), which is interesting, but obviously does not yet provide sufficient evidence for the reinstatement of Leucostola per se.

\section{Conclusions}

Our results provide first insights into an intrageneric structure within Argyra, which are largely consistent with morphological assumptions. In order to gain a better resolution that would allow a more reliable and overall picture of the phylogenetic relationships within Argyra, it is obvious that more species of Argyra (especially species with a pubescent scutellum, and a bare scape) from both the Palaearctic and Nearctic realms should be included in future analyses.

Acknowledgements. We thank Samuel Tanner (Zurich) for his support in the laboratory, and Christian Kropf and Stefan Hertwig (Natural History Museum, Bern) for the use of technical infrastructure of the museum. We are further thankful to Oleg Negrobov (Russia, Voronezh) for providing reprints, to Youna Zahn (Natural History Museum, Bern) for her steady help in finding literature, and to two anonymous referees for their valuable comments. This work has been supported by a grant of the Swiss National Science Foundation to M. V. Bernasconi (Grant 3100A0115981). The present paper is situated within the frame of the $\mathrm{PhD}$ thesis of the first author (Christoph Germann) and is a contribution of the Institute of Evolutionary Biology and Environmental Studies, Zoological Museum, University of Zurich, Switzerland.

\section{References}

Assis Fonseca, E. C. M. 1978: Diptera Orthorrhapha Brachycera Dolichopodidae. - Handbooks for the Identification of British Insects 9: 1-90.

Becker, T. 1918: Dipterologische Studien. Dolichopodidae. A. Paläarktische Region. - Nova Acta Academiae Caesareae Leopodinisch-Carolinae Germanicae Naturae Curiosorum 104: 35-214.

Bernasconi, M. V., Pollet, M., Varini-Ooijen, M. \& Ward, P. I. 2007a: Phylogeny of European Dolichopus and Gymnopternus (Diptera, Dolichopodidae) and the significance of morphological characters inferred from molecular data. - European Journal of Entomology 104: 601-617.

Bernasconi, M. V., Pollet, M., \& Ward, P. I. 2007b: Molecular systematics of Dolichopodidae (Diptera) inferred from COI and 12S rDNA gene sequences based on European exemplars. - Invertebrate Systematics 21: 453-470.

Germann, C., Pollet, M., Tanner, S., Backeljau, T. \& Bernasconi, M. V. 2010: Legs of deception: disagreement between molecular markers and morphology of long legged flies (Diptera, Dolichopodidae). — Journal of Zoological Systematics and Evolutionary Research 48: 238-247.

Kowarz, F. 1879: Die Dipteren-Gattungen Argyra Macq. und Leucostola Lw. - Verhandlungen der Kaiserlichköniglichen Zoologisch-Botanischen Gesellschaft in Wien 28: 437-462, 1 plate.

Loew, H. 1857: Neue Beiträge zur Kenntniss der Dipteren. Fünfter Beitrag. - Programm der Königlichen Realschule zu Meseritz (1857): 1-56.

Lundbeck, W. 1912: Diptera Danica. Genera and species of flies hitherto found in Denmark. Part IV. Dolichopodidae. - Gad, Copenhagen. 1-416.

Mik, J. 1878: Dipterologische Untersuchungen. — Jahresberichte des Kaiserlich-königlichen Akademischen Gymnasiums, Wien (1877/1878): 1-24.

Negrobov, O. P. 1991: Family Dolichopodidae. — In: Soos, A. \& Papp, L. (eds.), Catalogue of Palaearctic Diptera. Vol. 7. Dolichopodidae - Platypezidae. Akadémiai Kiadó, Budapest. pp. 11-139.

Parent, O. 1938: Diptères Dolichopodides. — Faune de France 35: 1-720.

Pollet, M. 1996: Systematic revision and phylogeny of the Palaearctic species of the genus Achalcus Loew (Diptera: Dolichopodidae) with the description of four new species. - Systematic Entomology 21: 353-386.

Pollet, M. 2007: Dolichopodidae. - In: Pape, T. (ed.), Fauna Europaea: Diptera, Brachycera. Fauna Europaea version 1.3. [www document] URL http://www. fauneur.org. (Site visited on 12 November 2009).

Pollet, M., Brooks, S. E. \& Cumming, J. M. 2004: Catalog of the Dolichopodidae (Diptera) of America north of Mexico. - Bulletin of the American Museum of Natural History 283: 1-114.

Pollet, M., Germann C., Tanner S., \& Bernasconi M. V. 2010: Hypotheses from mitochondrial DNA: congru- 
ence and conflicts with morphology in Dolichopodinae systematics (Diptera: Dolichopodidae). - Invertebrate Systematics 24: 32-50.

Posada, D. \& Crandall, K. A. 1998: Modeltest: testing the model of DNA substitution. - Bioinformatics 14: 817-818.

Ronquist, F. \& Huelsenbeck, J. P. 2003: MrBayes 3: Bayesian phylogenetic inference under mixed models. Bioinformatics 19: 1572-1574.

Selivanova, O. V. \& Negrobov, O. P. 2006a: The species of the genus Argyra Macquart (Diptera, Dolichopodidae) of the Far East and addition to the primary description of Argyra submontana Negrobov \& Selivanova. Bulletin of Moscow Society of Naturalists. Biological Series 111: 52-54.

Selivanova, O. V. \& Negrobov, O. P. 2006b: A new species of the genus Argyra from the Far East of Russia (Diptera: Dolichopodidae). - Zoosystematica Rossica 15: 169-170.

Selivanova, O. V. \& Negrobov, O. P. 2007: A new species of the genus Argyra Macquart from Voronezh Province of Russia (Diptera: Dolichopodidae). - Zoosystematica Rossica 16: 133-134.

Shiner, J. R. 1862: Fauna Austriaca. Die Fliegen (Diptera).
— Nach der analytischen Methode bearbeitet, mit der Karakteristik sämmtlicher europäischer Gattungen, der Beschreibung aller in Deutschland vorkommenden Arten und der Aufzählung aller bisher beschriebenen europäischen Arten. Wien. 674 pp.

Sinclair, B. J., Brooks, S. E. \& Cumming, J. M. 2008: A critical review of the world catalogues of Empidoidea (Insecta: Diptera) by Yang et al. $(2006,2007)$. — Zootaxa 1846: 61-68.

Swofford, D. L. 2002: PAUP*: Phylogenetic Analysis Using Parsimony (*and other methods), version 4.0 b10. - Sinauer Associates, Sunderland, MA.

Tamura, K., Dudley, J. Nei, M. \& Kumar, S. 2007: MEGA4: Molecular Evolutionary Genetics Analysis (MEGA) software version 4.0. - Molecular Biology and Evolution 24: 1596-1599.

Van Duzee, M. C. 1925: A revision of the North American Species of the Genus Argyra, two-winged Flies of the Family Dolichopodidae. — Proceedings of the United States National Museum 66: 1-43.

Yang, D., Zhu, Y., Wang, M. \& Zhang, L. 2006: World Catalog of Dolichopodidae (Insecta: Diptera) - China Agricultural University Press, Beijing, Beijing. 704 pp. 